\title{
Educação e inclusão digital para idosos*
}

Michele Marinho da Silveira - Fisioterapeuta graduada pela Universidade de Passo Fundo; Pós-graduada em Ortopedia e Traumatologia pelo Colégio Brasileiro de Estudos

Sistêmicos; Mestranda bolsista do Programa de Pós-Graduação em Envelhecimento

Humano da Universidade de Passo Fundo. E-mail: mm.silveira@ yahoo.com.br. Josemara de Paula Rocha - Fisioterapeuta graduada pela Universidade de Passo Fundo, Mestranda bolsista do Programa de Pós-Graduação em Envelhecimento Humano da Universidade de Passo Fundo. E-mail: josemara.rocha@hotmail.com.

Marlon Francys Vidmar - Acadêmico do curso de Fisioterapia da Universidade de Passo Fundo. Bolsista Pibic/UPF. E-mail: marlonfrancys@msn.com.

Lia Mara Wibelinger - Docente do curso de Fisioterapia da Universidade de Passo Fundo; Mestre e doutoranda em Gerontologia Biomédica pela Pontifícia Universidade Católica do Rio Grande do Sul. E-mail: liafisio@upf.br.

Adriano Pasqualotti - Professor Titular da Universidade de Passo Fundo. Coordenador do Programa de Pós-Graduação em Envelhecimento Humano da Universidade de Passo Fundo. Doutor em Informática na Educação e Mestre em Ciência da Computação pela Universidade Federal do Rio Grande do Sul. Editor da Revista Brasileira de Ciências do Envelhecimento Humano. E-mail: pasqualotti@upf.br

Endereço para correspondência: Universidade de Passo Fundo, Faculdade de Educação Física e Fisioterapia. BR 285, km 171, Bairro São José, Cx. Postal 611, CEP 99100000, Passo Fundo - RS, Brasil.

Resumo

A dificuldade em entender a nova linguagem tecnológica traz consigo um problema social; e o idoso, por vezes, retorna ao caminho da educação na perspectiva de uma atualização cultural e reaproximação social. As tecnologias de informação e comunicação intensificarão esse processo de aprendizagem, ao permitir interagir com

\footnotetext{
* Estudo bibliográfico realizado na disciplina Escola e Qualidade de Vida do Programa de Pós-Graduação em Envelhecimento Humano da Universidade de Passo Fundo.
} 
diferentes informações, pessoas e grupos, e socializar seus conhecimentos e suas próprias histórias de vida, aumentando sua auto-estima e auto-realização. Este artigo tem como objetivo discutir algumas questões importantes para uma educação gerontológica mais participativa e transformadora, desde as manifestações clínicas do envelhecimento, a possibilidade de inclusão digital e a educação gerontológica mediada pela informática.

Palavras-chaves: Gerontologia. Educação. Tecnologia. Inclusão. Idoso.

Education and digital inclusion for elderly

\section{Abstract}

The difficulty in understanding the new language of technology brings with it a social problem; and the elderly sometimes return to the path of education from the perspective of an upgrade cultural and social rapprochement. Information technology and communication will enhance the learning process by allowing interaction with different information, people and groups, and socialize their knowledge and their own stories of life, increasing their self-esteem and self-realization. This article aims to discuss some important issues for gerontological education more participatory and transformer, since the clinical manifestations of the aging, the possibility of digital inclusion and a gerontological education mediated by computer.

Key-words: Geriatrics. Education. Technology. Inclusion. Elderly.

Introdução

Estudos demográficos nacionais realizados pelo Instituto Brasileiro de Geografia e Estatística (IBGE) demonstram que o envelhecimento da população não é apenas uma característica dos países desenvolvidos. A parcela da população brasileira com mais de sessenta anos de idade cresceu de 4\% em 1940 para 10\% na atualidade, sendo que a 
população de idosos representa um contingente de quase 15 milhões de pessoas com sessenta anos ou mais de idade (8,6\% da população brasileira).

Diante disso, a realização de políticas públicas e estudos que contribuam para a qualidade de vida na terceira idade validam-se não apenas na valorização da dignidade do idoso enquanto cidadão, mas também na medida em que satisfazem as necessidades de uma parcela significativa da população, por isso a tecnologia e a educação irão agir juntas para evitar que esse contingente de pessoas fique a margem da sociedade. (Warschauer, 2006). Em relação à educação, as propostas educacionais alinhadas com a segregação dos idosos mostram-se agora em vias de falência, diante da heterogeneidade de experiências de velhice que se vê no Brasil de hoje, exigindo ações educativas diferenciadas no campo da gerontologia. (Netto, 2002).

Os adultos maduros e idosos que atualmente retomam o caminho da educação o fazem não apenas pela perspectiva da atualização cultural, da busca por novos vínculos sociais, necessidades de regulação emocional ou como atividades para ocupar o tempo livre. Procuram atividades para desenvolvimento de habilidades específicas, para uso prático dos conhecimentos. Procuram espaços educativos mais críticos e contextualizados. (Leão, 2008).

Além disso, a tecnologia tem o poder de facilitar a vida de alguns indivíduos e na mesma proporção pode penalizar determinados grupos da população. Vale ressaltar que uma parcela dessa população, que atualmente vem sofrendo algumas restrições com os avanços tecnológicos, são os idosos. A atual geração de idosos tem revelado dificuldades em entender a nova linguagem tecnológica e em lidar com esses avanços até na realização de tarefas básicas como, por exemplo, operar eletrodomésticos, celulares e caixas eletrônicos instalados nos bancos. (Nogueira et al., 2008).

Segundo Roldão (2009) a aprendizagem contínua é fundamental e após revisão literária, cita que neste contexto há cinco caminhos no qual a qualidade de vida pode estar intrincada nesse processo. Por possibilitar uma compreensão atualizada do seu meio sociocultural; conscientizar sobre suas próprias potencialidades na velhice; protegê-lo quanto à saúde exercitando o cérebro; possibilitando atividades e espaços para formação da personalidade; ao ser organizado em atividade grupal, o contato com outras pessoas pode favorecer a adoção de condutas resilientes. 
As universidades têm oferecido projetos de extensão, e a integração do idoso é uma questão de livre escolha do mesmo, mas o mesmo deve ter o acesso a essas oportunidades. Este texto tem como objetivo discutir algumas questões que norteiam o envelhecimento para uma educação gerontológica mais participativa e transformadora, desde as manifestações clínicas do envelhecimento, a possibilidade de inclusão digital e a educação gerontológica mediada pela informática.

Manifestações clínicas do envelhecimento

É notório o rápido crescimento do tempo de vida da população brasileira nas últimas décadas. O índice de envelhecimento da população, que era igual a 6,4 em 1960, alcançou 13,9 em 1991, representando 7,5\% da população de idosos em todo o mundo, o que demandará custos com políticas públicas de saúde. (Chaimowicz, 1997).

No Brasil considera-se idoso a pessoa que tem sessenta anos ou mais de idade, é uma fase da vida em que as pessoas tiveram muito ganhos, mas também muitas perdas, entre as quais, destaca-se a saúde como um dos aspectos mais afetados nos idosos. (Rodrigues; Diego, 1996). Porém, o envelhecimento não pode ser visto como uma mera passagem de tempo, mas a manifestação de eventos biológicos que ocorrem ao longo de um período representando perdas da função normal ao longo da vida (Hayflick, 1997).

E mais que isso, o envelhecimento pode ser conceituado como um processo dinâmico e progressivo, onde há alterações morfológicas, biológicas, funcionais e químicas que alteram progressivamente o organismo, tornando-o sensível a agressões internas e externas, (Netto, 2002), mas que manifestam ritmo, duração e efeitos com diferenças individuais, entre grupos etários, dependentes de eventos e natureza genéticobiológica, sócio-histórica e psicológica. (Néri, 2001).

E todos os processos de transformação do organismo, tanto físicos quanto psicológicos e sociais, acabam envolvendo principalmente os papeis sociais desempenhados pelos indivíduos. (Passerino; Pasqualotti, 2006).

Segundo Thompson, Skinner e Piercy (2002), os sinais do envelhecimento vão aparecendo com a idade, incluem-se neles: branqueamento e espessamento do cabelo, perda de elasticidade e secura da pele, audição pode ficar prejudicada, visão diminuída e adaptação ao escuro limitada, a fala pode tornar-se restrita, o aprendizado e a memória à curto prazo ficam prejudicados, os limiares da dor são altos e a sensibilidade a ela 
diminui, o sistema cardiovascular diminui quanto a eficiência, ocorre uma menor mobilidade torácica, diminuição da elasticidade dos tecidos moles, as articulações absorvem menos pressões e são mais rígidas, as cartilagens são menos elásticas, o poder muscular diminui levando a desaceleração dos movimentos, perda da coordenação, dificuldades com o equilíbrio e os ossos podem se tornar osteoporóticos.

Todas essas manifestações vão influenciar diretamente nas habilidades motoras para se fazer uso dos dispositivos tecnológicos e estes precisam estar adaptados para os idosos que apresentam movimentos lentificados, menor acuidade visual e auditiva, por exemplo.

Inclusão digital para idosos

Envelhecer, por muito tempo significou viver excluído da sociedade e ser um peso para a família. Nos últimos anos, com o avanço da ciência e da medicina, esta etapa da vida começa a ser vivida com mais qualidade. Além disso, alguns mitos referentes ao envelhecimento vem sendo quebrados. Conforme Kachar (2001), o perfil do idoso do século XXI mudou, ele deixou de ser uma pessoa que vive de lembranças do passado, recolhido em seu aposento, para uma pessoa ativa, capaz de produzir, participante do consumo, que intervém nas mudanças sociais e políticas.

Assim, como o corpo deve ser exercitado para prolongar a vida e a saúde, há alguns anos descobriu-se que a atividade mental pode modificar o comportamento acomodado que alguns idosos adotam ao envelhecer. (Vergara, 1999).

Essa geração que nasceu e foi educada em uma época em que o tempo transcorria em outra velocidade e as tendências das situações eram a estabilidade, hoje não consegue acompanhar as modificações sociais e tecnológicas. Para a maioria dos idosos, o uso do computador estaria totalmente fora do seu alcance, não envolvendo apenas motivos financeiros, mas emocionais. O uso desta tecnologia traz certas dificuldades que para nós passam despercebidas, tudo é muito desconhecido: os ícones, o mouse, a velocidade, dificuldade em ler na tela, o peso dos dedos sobre o teclado, a memória, a coordenação visomotora, e visão frágil para visualizar os ícones pequenos. (Kachar, 2000).

$\mathrm{O}$ avanço da tecnologia, somado às dificuldades de acesso às novas técnicas e teorizações, causa impacto em todas as gerações e, em especial, na velhice. Bens 
tecnológicos de ultima geração contrastam com a miséria, pois o não-acesso a esses bens remete à exclusão e ao isolamento social. Entretanto a mídia já consegue identificar o envelhecimento como um novo mercado de consumo, criando e divulgando novos mecanismos de educação e atualização na internet, capazes de oferecer respostas criativas ao conjunto de mudanças sociais que redefinem a experiência do envelhecimento como uma fase de conquista coletiva. (Pasqualotti, 2003).

Se por um lado, as novas gerações apresentam familiaridade com o uso das inovações tecnológicas que surgem aceleradamente as gerações mais velhas, dos idosos, por sua vez, encontram-se no extremo oposto, sentindo-se no meio de um "bombardeio tecnológico" que lhes causa estranheza, medo e/ou receio. Essa geração sente-se analfabeta diante das novas tecnologias, revelando dificuldades em entender a nova linguagem e em lidar com os avanços tecnológicos, até mesmo em questões básicas com eletrodomésticos, celulares e os caixas eletrônicos instalados nos bancos. (Kachar, 2003).

As pessoas acima de cinquienta anos, por exemplo, apesar de possuírem um acúmulo de experiências, que só o viver propicia e, apesar de ainda estarem trabalhando, costumam sofrer preconceitos no local de trabalho no que diz respeito a sua eficiência num mundo tecnológico, pois a relação com a tecnologia é vista como um indicador de eficiência, portanto a sua é posta em dúvida. Esse preconceito e a pressão social e profissional para que pessoas mais velhas se apropriem das novas tecnologias como os mais jovens fazem com que algumas delas entrem em um novo movimento rumo a uma maior familiarização tecnológica, como constata Kachar (2003).

Conforme Alves (2008), a inclusão digital é a democratização do acesso ao mundo da informática que com o avanço das tecnologias surgiu um novo tipo de analfabetismo, o analfabetismo digital.

$\mathrm{Na}$ questão da inclusão digital do idoso na utilização das tecnologias de informação e comunicação devemos estar atentos aos obstáculos que se interpõem entre o idoso e a tecnologia. Muitas vezes argumenta-se que o idoso não se apropria corretamente da tecnologia em razão da velocidade vertiginosa com que muda e, pela angustia resultante desse movimento tecnológico, acaba desistindo da inclusão. (Passerino; Pasqualotti, 2006). 
Conforme afirma Kachar (2003), diferentemente dos sujeitos que já nasceram durante o surgimento e ascensão das tecnologias digitais, a geração de tempos de relativa estabilidade tem uma convivência conflituosa em meio às complexas e rápidas mudanças tecnológicas. Assim, compreendemos que o idoso apresenta necessidades educacionais especiais no que concerne à aprendizagem sobre as tecnologias, pois, conforme Vygotsky (1998), o desenvolvimento de um sujeito não pode ser compreendido por meio de um estudo do indivíduo. É necessário considerar também o mundo social externo no qual aquela vida individual desenvolve-se, de maneira que, o contexto social e histórico no qual os idosos contemporâneos desenvolveram-se não estava imbuído da tecnologia.

A inclusão, então, é um processo a partir do qual uma pessoa ou grupo de pessoas passa a participar de usos e costumes de outro grupo e ter os mesmos direitos e deveres daqueles; a inclusão digital é vista como uma forma de inclusão social, porque por meio das tecnologias de informação e comunicação é possível a participação na sociedade através de outras vias de acesso e pelo desenvolvimento social, cognitivo e afetivo que podem promover nos sujeitos. (Passerino; Pasqualotti, 2006).

Mesmo com todo avanço tecnológico ocorrido, ainda existem pessoas que não sabem utilizar a multiplicidade de serviços oferecidos no mundo virtual, fazendo com que a preocupação com a inclusão digital de idosos seja cada vez maior. O processo de inclusão digital proporciona aos idosos a recuperação da auto-estima, o exercício da cidadania e interação social.

Educação em gerontologia mediada pela informática

A gerontologia é vasto campo profissional e disciplinar, do qual faz parte a educação gerontológica, que se ocupa da educação dos idosos e da formação de profissionais para esse atender este público. No Brasil, a construção da educação gerontológica avança principalmente a partir da atuação de cursos de pós-graduação em gerontologia; é fortalecida pela criação de universidades da terceira idade, importante lócus de programas para idosos, de pesquisa e de formação de recursos humanos, embora nelas ainda predominem ações pedagógicas não-especializadas. (Cachioni; Néri, 2004). 
A preocupação com a qualidade de vida e a educação permanente das pessoas idosas tem levado os centros universitários que desenvolvem programas de extensão a implementarem programas voltados especificamente para esse segmento da população. (Pasqualotti, 2004).

Um exemplo de educação gerontológica que existe há mais de 10 anos é o CREATI* da Universidade de Passo Fundo, que através das oficinas de informática os idosos aprendem a utilizar o computador para desenvolver o raciocínio e a lógica, enquanto interagem com os colegas, familiares e amigos das mais diferentes faixas etárias. Cada idoso trabalha individualmente no seu computador, acompanha as tarefas através do uso do canhão de projeção e os computadores têm acesso à internet. Os idosos aprendem a manusear o mouse e o teclado, e posteriormente são levados a cadastrar e a manter o seu próprio e-mail. (Oficina de Informática do CREATI, 2010).

Assim, no âmbito pedagógico, constata-se a existência de espaços para avanços conceituais que transcendam os referenciais de qualidade da educação permanente, incorporando à prática pedagógica a possibilidade de qualificar dimensões relativas à saúde física, emocional, espiritual, ambiental e à experiência de um convívio social fundamentado em crenças pessoais e em valores condizentes com uma aprendizagem emancipatória, promotora da cidadania e da ética. A educação para saúde e a qualidade de vida na velhice é questão emergente na atualidade, até porque a própria demografia está a exigir. (Pasqualotti, 2004).

Um trabalho executado pelo idoso com auxílio do computador é enriquecedor pelo fato de oportunizar a construção de conhecimento e a aprendizagem contínua, despertando seu interesse e pensamento crítico. (Pasqualotti, 2004).

Para Kachar (2001), o advento da tecnologia provê para a pessoa da terceira idade oportunidades para se tornar um aprendiz virtual, fornecendo educação continuada, educação à distância, estimulação mental e bem-estar. A tecnologia possibilita ao indivíduo estar mais integrado em uma comunidade eletrônica ampla; coloca-o em contato com parentes e amigos, num ambiente de troca de idéias e informações, aprendendo junto e reduzindo o isolamento por meio da experiência comunitária.

\footnotetext{
* Centro Regional de Estudos e Atividades para a Terceira Idade

V. $8 \mathrm{~N}^{\mathrm{o}} 2$, julho, 2010
} 
É relevante investigar quais as abordagens adequadas para introduzir o idoso no universo da informática e construir estratégias metodológicas educacionais para preparar os idosos (ativos ou aposentados) no domínio operacional dos recursos computacionais. É necessário gerar a alfabetização na nova linguagem tecnológica que se instala em todos os setores da sociedade e promover a inclusão do idoso nas transformações da sociedade. A abordagem educacional com idosos tem suas peculiaridades e requer a imersão neste universo para compreendê-lo e uma prática pedagógica específica, considerando as características físicas, psicológicas e sociais dessa faixa etária. (Kachar, 2001).

A introdução do computador na educação tem provocado uma verdadeira revolução na concepção de ensino e aprendizado. Em primeiro lugar, as análises de programas que agem no processo ensino-aprendizagem mostram que eles não podem ser caracterizados como uma mera versão computadorizada dos atuais métodos de ensino; e em segundo lugar, as novas modalidades do uso do computador na educação não podem ser caracterizadas apenas como "máquinas de ensinar", mas como uma nova ferramenta educacional de complementação, de aperfeiçoamento e de possível mudança na qualidade do ensino-aprendizagem. (Pasqualotti, 2003). Um exemplo de ambiente que viabiliza a socialização do conhecimento e o surgimento de idéias elaboradas através da interação numa rede social é a ferramenta InterDigital Arte. A característica interativa da ferramenta permite ao sujeito ser autor ou co-autor de pintura de cenários. A interação possibilita a criação de forma colaborativa de objetos que podem ser incorporado ao ambiente num movimento contínuo e recursivo. (Pasqualotti, 2008).

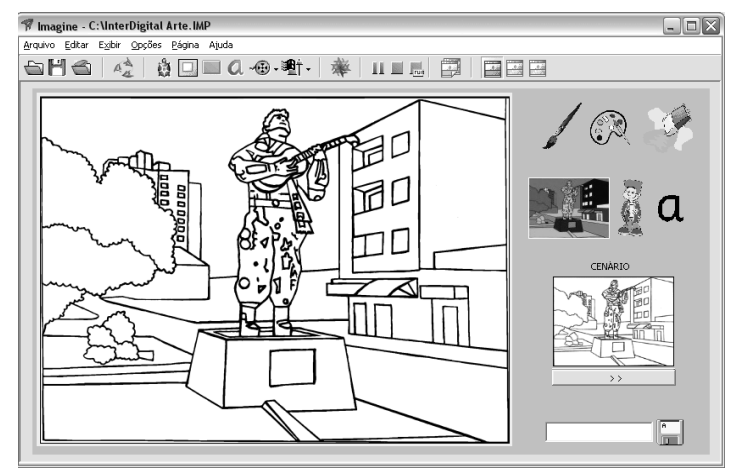

Figura 1. Ferramenta InterDigital Arte para Pintura de Cenários no Ciberespaço. 
O portal InterDigital (www.interdigital.org.br) integra as ferramentas de comunicação e interação que foram desenvolvidas com a finalidade de permitir o estabelecimento de relações produtivas e a construção da identidade social que estão sendo desenvolvidas. (Pasqualotti, 2008).

Conclusão

A partir dessa reflexão podemos verificar que a importância da inclusão digital na educação continuada, a preocupação desta com a qualidade de vida e a interação social dos idosos vem sendo discutida num contexto bastante amplo do envelhecimento. As leituras realizadas permitiram visualizar que uma educação ao longo da vida, utilizando as tecnologias de informação e comunicação, parece ser uma alternativa para que os idosos continuem a aprender e usufruir o seu tempo, estimulando-os a aprender usar o computador em rede, abrindo novas possibilidades e oportunidades. Notou-se, também, a carência de estudos que contribuam para a realização de uma política pública focada à inclusão digital como uma estratégia metodológica educacional.

Referências Bibliográficas

ALVES, Luciana. Programa um Computador Para Todos. s.d. Disponível em: <http://www.computadorparatodos.gov.br> Acesso em: 10 de mai. de 2010.

CACHIONI, M; NÉRI, A. L. Educação e Gerontologia: Desafios e Oportunidades. Revista Brasileira de Ciências do Envelhecimento Humano, Passo Fundo, v. 1, n. 1, p. 99-115, jan./jun. 2004

CHAIMOWICZ, Flávio. A saúde dos idosos brasileiros às vésperas do século XXI: problemas projeções e alternativas. Revista Saúde Pública, São Paulo, v. 31, n. 2, p. 184-200, abr. 1997. 
DIOGO, M. J. D.; DUARTE, Y. A de O. O envelhecimento e o idoso no ensino de graduação em enfermagem no Brasil: do panorama atual a uma proposta de conteúdo programático. Revista da Escola de Enfermagem, São Paulo, v. 33, n. 4, p. 370-376, 1999.

HAYFLIK, Leonard. Como e Por Que Envelhecemos. Rio de Janeiro: Campus, 1997.

IBGE. Perfil dos Idosos Responsáveis pelos Domicílios. Disponível em:<http://www.ibge.gov.br/home/presidencia/noticias/25072002pidoso.shtm>. Acesso em: 10 de maio. 2010.

KACHAR, Vitória. A terceira idade e o computador: interação e transformações significativas. A Terceira Idade, São Paulo, v. 11, n. 19, p. 5-21, 2000.

KACHAR, Vitória. A Terceira Idade e o Computador: Interação e Produção no Ambiente Educacional Interdisciplinar. São Paulo: PUC/SP, 2001. 206p. Tese de Doutorado em Educação.

KACHAR, Vitória. Terceira Idade \& Informática: Aprender revelando potencialidades. São Paulo: Cortez, 2003.

LEÃO, Marluce Auxiliadora Borges Glaus. Educação permanente de adultos maduros, idosos e de profissionais da área do envelhecimento: fundamentos para um projeto pedagógico de extensão universitária. Revista de Extensão da Universidade de Taubaté - Pró-Reitoria de Extensão e Relações Comunitárias, Taubaté, n. 1, p. 45$54,2008$.

NETTO, Matheus Papaléo. Gerontologia: A Velhice e o Envelhecimento em Visão Globalizada. São Paulo: Atheneu, 2002, 524 p. 
NERI, Anita Liberalesso. Palavras-Chave em Gerontologia. Campinas: Alínea, 2001.

NOGUEIRA, N. P. et al. Inclusão Digital do Idoso. In: $\underline{\text { XIX SIMPÓSIO }}$ BRASILEIRO DE INFORMÁTICA NA EDUCACC̃̃O, 20, 2008, Fortaleza. Anais. Fortaleza: Núcleo de projeto em tecnologia da informação/Universidade Estadual do Ceará, 2008.

OFICINA DE INFORMÁTICA DO CREATI (Centro Regional de Estudos e Atividades para a Terceira Idade). Coordenação de Cristiane Carla Schenkel. Desenvolvido pela Universidade de Passo Fundo. 1991-2010. Apresenta diversas oficinas para a terceira idade. Disponível em: http://www.upf.tche.br/creati/c_informatica.html. Acesso em: 10 de Mai. De 2010.

PASQUALOTTI, Adriano. Desenvolvimento dos aspectos sociais na velhice: experimentação de ambientes informatizados. In: BOTH, A.; BARBOSA, M. H.; BENINCÁ, C. R. (Orgs.). Envelhecimento Humano: Múltiplos Olhares. Passo Fundo: Universidade de Passo Fundo, 2003, p. 39-56.

PASQUALOTTI, A. et al. Experimentação de ambientes informatizados para pessoas idosas: avaliação da qualidade de vida. In: Workshop de Computação da Região Sul, 2004, Florianópolis, Anais... Florianópolis: Universidade de Santa Catarina, 2004.

PASSERINO, L. M.; PASQUALOTTI, P. R. A inclusão digital como prática social: uma visão sócio-histórica da apropriação tecnológica em idosos. In: PORTELLA, M. R.; PASQUAlotTI, A., GAGLIETTI, M. (Orgs.). Envelhecimento Humano: Saberes e Fazeres. Passo Fundo: Universidade de Passo Fundo, 2006, p. 246-260. 
PASQUALOTTI, A. Comunicação, tecnologia e envelhecimento: significação da interação na era da informação, no Estado do Rio Grande do Sul. Porto Alegre: UFRGS, 2008. 198p. Tese de Doutorado em Informática na Educação.

ROLDÃO, Flávia Diniz. Aprendizagem Contínua de Adulto-Idosos e Qualidade de Vida: Refletindo sobre Possibilidades em Atividades de Extensão nas Universidades. Revista Brasileira de Ciências do Envelhecimento Humano, Passo Fundo, v. 6, n. 1, p.6 1-73, jan./abr. 2009.

THOMSON, A.; SKINNER, A.; PIERCY, J. Fisioterapia de Tydi. $12^{\mathrm{a}}$ ed. São Paulo: Santos, 2002.

VERGARA, R. e FLORESTA, C. Idosos no Brasil estão cada vez mais ativos. Folha de São Paulo, São Paulo, 06 ago. 1999. 2. cad. p. 5.

VYGOTSKY, Lev Semenovich. A Formação Social da Mente: o desenvolvimento dos processos psicológicos superiores. Trad. José Cipolla Neto, Luís Silveira Menna Barreto, Solange Castro Afeche. $6^{\text {a }}$ ed. São Paulo: Martins Fontes, 1998, 191 p.

WARSCHAUER, Mark. Tecnologia e Inclusão Social: a Exclusão Digital em Debate. São Paulo: Senac, 2006. 319p. 